\title{
Phase Transition of the Horava-Lifshitz AdS Black Holes
}

\author{
Yun-Zhi Du ${ }^{1,2}$ (D) Hui-Hua Zhao ${ }^{1,2} \cdot$ Li-Chun Zhang ${ }^{1,2}$
}

Received: 23 January 2021 / Accepted: 14 April 2021 / Published online: 28 April 2021

(C) The Author(s) 2021

\begin{abstract}
Some ones have showed the first-order phase transition of the Horava-Lifshitz (HL) AdS black holes has unique characters from other AdS black holes. While the coexistence zone of the first-order phase transition was not exhibited. As well known the coexistence curve of a black hole carries a lot of information about black hole, which provides a powerful diagnostic of the thermodynamic properties on black hole. We study the first-order phase transition coexistence curves of the HL AdS black holes by the Maxwell's equal-area law, and give the boundary of two-phase coexistence zone. It is very interesting that the firstorder phase transition point is determined by the pressure F on the surface of the HL AdS black hole's horizon, instead of only the pressure $\mathrm{P}$ (or the temperature T). This unique property distinguishes the HL AdS black hole from the other AdS black hole systems. Furthermore, this black hole system have the critical curves, and on which every point stands for a critical point.
\end{abstract}

Keywords Phase transition · Horava-Lifshitz AdS black hole · Equal-area law

\section{Introduction}

Recently, people pay more attention to the phase transition of Anti-de-Sitter (AdS) and deSitter (dS) black holes by regarding the cosmological constant of a n-dimensional AdS black hole $\Lambda=-\frac{n(n-1)}{2 l^{2}}$ as the pressure $P=\frac{n(n-1)}{16 \pi l^{2}}$. Especially the critical phenomena of phase transition of a AdS black hole in the P-V diagram were investigated in Refs. [1-23]. The

Li-Chun Zhang

zhao2969@sina.com

Yun-Zhi Du

duyzh13@1zu.edu.cn

1 Department of Physics, Shanxi Datong University, Datong 037009, China

2 Institute of Theoretical Physics, Shanxi Datong University, Datong 037009, China 
research of the phase transition will not only help us to understand the nature of black holes more deeply, but also understand some phase transition behaviors in conformal field. The authors in Ref. [24] found when choosing $Q^{2}-\Psi$ as the independent dual parameters, the charged AdS black hole has the similar phase transition to that in the van der Waals (vdW). Furthermore, people found that for the more parameters black hole, the behavior of phase transition is similar to that of $\mathrm{vdW}$ by adopting the different independent dual parameters $[15,25-36]$. And the entanglement entropy in the AdS black holes also has the similar vdW's Phase Transition [37-44]. The authors investigated the phase transition of a AdS black hole by the Maxwell's equal area law and the adoption of independent dual parameters $T-S$, and found the phase transition point of the entanglement entropy is consistent with the first-order phase transition point. Moreover there exists one kind of black holes, whose has the three-phase coexistence point with similar vdW's system [4, 7, 8, 25, 45-48].

In Refs. [49-51], the $C_{P}-T$ curve near the critical point of the LoveLock AdS black holes and the Horava-Lifshitz (HL) AdS black holes is consistent with the $C_{V}-T$ curve of ${ }^{4} \mathrm{He}$. As what we have known, there exists the $\lambda$ phase transition as ${ }^{4} \mathrm{He}$ into the superfluid state. And the reasonable physical explanation have been given: the $\lambda$ phase transition may be a Bose condensation, and superfluid is related to the Bose body condensed at zero energy level. Therefore, the physical explanation of the similar phase transition of black holes is also an interesting issue. In order to give the corresponding physical explanation of phase transition, we should explore more thermodynamical properties of black holes deeply. The HL gravity, which is proposed by Horava, is a power-counting renormalizable gravity theory and can be regarded as an ultraviolet complete candidate for general relativity [52, 53]. And the black hole solutions, thermodynamics and phase transitions of the HL black hole have attracted a lot of attention [54-59]. People have given the condition of second-order phase transition and the critical exponents of HL AdS black holes by investigating the P-V diagram. Note that the first-order phase transition point of HL AdS black holes is a curve with a certain condition, and the $C_{P}-T$ curve near the second-order point is similar the $\lambda$ phase transition.

From the classification of the phase transition by Ehrenfest, we know that there are the obviously difference between the first-order phase transition and the second-order $\lambda$ one. For the liquid-gas phase transition in a vdW system, the $\lambda$ phase transition has the obvious signal: the heat capacity has a sharp increase before reaching the critical temperature, while the divergence of the first-order phase transition occurs when two phases coexist. The firstorder phase transition will be transformed to the second-order one as $T \rightarrow T_{c}\left(T<T_{c}\right)$. For example, when the liquid-gas phase transition $T<T_{c}$, the entropy is discontinuity. And this discontinuity will be more and more small as $T \rightarrow T_{c}$, until it becomes zero for $T=T_{c}$ or $P=P_{c}$. At the same time, there is a vertical slope in the T-S diagram, that is the corresponding $\lambda$ phase transition. Therefore, there are some questions naturally: whether there is the vdW-like first-order phase transition for the HL AdS black holes? If the answer is yes, what is the condition and the corresponding physical reason of the phase transition for the HL AdS black holes?

In this paper, the first-order phase transition of the four-dimensional HL AdS black holes thermodynamic system is explored by Maxwell's equal area law. We will exhibit the the condition of the two phases coexisting by adopting the independent dual parameters T-S and give the corresponding physical explanation. Furthermore the factors, which will affect the coexistence zone of the first-order phase transition, are also analyzed. 


\section{Thermodynamic Quantities of Horava-Lifshitz Black Holes}

In this section, we will present the extended thermodynamics of the generalized topological HL black holes. The action of the HL gravity without the detailed-balance condition is [53]:

$$
I=\int d t d^{d} x\left[L_{0}+\left(1-\epsilon^{2}\right) L_{1}+L_{m}\right]
$$

with the Lagrangian of other matter fields $L_{m}$ and

$$
\begin{aligned}
& L_{0}=\sqrt{g} N\left[\frac{2}{k^{2}}\left(K_{i j} K^{i j}-\lambda K^{2}\right)+\frac{k^{2} \mu^{2}\left[(d-2) \Lambda R-d \Lambda^{2}\right]}{8(1-d \lambda)}\right], \\
& L_{1}=\sqrt{g} N\left[\frac{k^{2} \mu^{2} R^{2}}{8(1-d \lambda)}\left(1-\frac{d}{4}-\lambda\right)-\frac{k^{2}}{2 \omega^{4}} Z_{i j} Z^{i j}\right] .
\end{aligned}
$$

Here $Z_{i j}=C_{i j}-\frac{\mu \omega^{2}}{2} R_{i j}$ with the Cotton tensor $C_{i j}$. In this theory, there are several parameters: $\epsilon, k^{2}, \lambda, \mu, \omega$ and $\Lambda$.

Compared with the general relativity, there are the relations for the parameters:

$$
c=\frac{k^{2} \mu}{4} \sqrt{\frac{\Lambda}{1-d \lambda}}, \quad G=\frac{k^{2} c}{32 \pi}, \quad \bar{\Lambda}=\frac{d \Lambda}{2(d-2)},
$$

where $c, G$ and $\bar{\Lambda}$ are Newton's constant, speed of light and consmological constant, respectively. We will fix $\lambda=1$ in the following, only for which the general relativity can be reconvered in the large distance approximation. In addition, we will only consider the general values of $\epsilon$ with the region $0 \leq \epsilon^{2} \leq 1$.

In this system there are the arbitrary dimensional topological AdS black holes with the metric [50]:

$$
d s=-f(r) d t^{2}+f^{-1}(r) d r^{2}+r^{2} d \Omega_{d-1, k}^{2}
$$

with

$$
\begin{aligned}
f(r)= & k+\frac{32 \pi P r^{2}}{\left(1-\epsilon^{2}\right) d(d-1)}-4 r^{2-d / 2} \\
& \times \sqrt{\frac{(d-2) M P \pi}{d\left(1-\epsilon^{2}\right)}+\frac{64 \epsilon^{2} P^{2} \pi^{2} r^{d}}{d^{2}\left(1-\epsilon^{2}\right)^{2}(d-1)^{2}}} .
\end{aligned}
$$

Here $d \Omega_{d-1, k}^{2}$ denotes the line element of a (d-1)-dimensional manifold with the constant scalar curvature $(d-1)$, and $k=0, \pm 1$ indicate different topology of the spatial spaces. In AdS spacetime, the cosmological constant is introduced as the thermodynamical pressure [1]: $P=-\frac{\Lambda}{8 \pi}$

The mass of this system reads

$$
M=\frac{64 \pi P r_{+}^{d}}{d(d-1)^{2}(d-2)}+\frac{1-\epsilon^{2} d k^{2} r_{+}^{d-2}}{16 P \pi(d-2)}+\frac{4 k r_{+}^{d-2}}{(d-1)(d-2)},
$$

where $r_{+}$denotes the event horizon which the largest positive root of $f\left(r_{+}\right)=0$. The conjugate thermodynamic volume of pressure, the entropy and temperature are presented 
[28] in the following forms:

$$
\begin{aligned}
V= & \frac{64 \pi r_{+}^{d}}{d(d-1)^{2}(d-2)}-\frac{\left(1-\epsilon^{2}\right) d k^{2} r_{+}^{d-4}}{16 P^{2} \pi(d-2)}, \\
S= & \left\{\begin{array}{l}
4 \pi r_{+}^{2}\left(1+\frac{3 k\left(1-\epsilon^{2}\right) \ln r_{+}}{8 \pi P r_{+}^{2}}\right)+S_{0} \\
\frac{16 \pi r_{+}^{d-1}}{(d-1)^{2}(d-2)}\left(1+\frac{k d(d-1)^{2}(d-2)\left(1-\epsilon^{2}\right)}{32(d-2)(d-3) P \pi r_{+}^{2}}+S_{0}\right) d \geq 4
\end{array},\right. \\
T= & \frac{1}{8(d-1) \pi r_{+}\left[32 \pi r_{+}^{2} P+k d(d-1)\left(1-\epsilon^{2}\right)\right]} \\
& \times\left\{1024 P^{2} \pi^{2} r_{+}^{4}+64 k(d-1)(d-2) P \pi r_{+}^{2}\right. \\
& \left.\quad+k^{2} d(d-1)^{2}(d-4)\left(1-\epsilon^{2}\right)\right\} .
\end{aligned}
$$

It is easy to check the first law of thermodynamics as

$$
d M=T d S+V d P+\Psi d \epsilon^{2}
$$

with the potential

$$
\Psi=-\frac{d k^{2} r_{+}^{d-4}}{16 P \pi(d-2)} .
$$

\section{The Construction of the Equal-Area Law in $T-S$ Diagram}

For the HL AdS black hole thermodynamic system with the unchanged pressure in the equilibrium state, the entropies at the boundary of the two-phase coexistence area are $S_{1}$ and $S_{2}$, respectively. And the corresponding temperature is $T_{0}$, which is less than the critical temperature $T_{c}$ and is determined by the horizon radius $r_{+}$. Therefore, from the Maxwell's equal-area law $T_{0}\left(S_{2}-S_{1}\right)=\int_{S_{1}}^{S_{2}} T d S$, we have in the four-dimensional spacetime $(d=3)$

$$
\begin{aligned}
0= & T_{0}\left(4 \pi r_{2}^{2}\left(1-x^{2}\right)-\frac{3 k\left(1-\epsilon^{2}\right) \ln x}{2 P}\right)-2 k r_{2}(1-x) \\
& -\frac{16}{3} P \pi r_{2}^{3}\left(1-x^{3}\right)+\frac{3 k^{2}\left(1-\epsilon^{2}\right)(1-x)}{16 \pi P r_{2} x}
\end{aligned}
$$

with $x=\frac{r_{1}}{r_{2}}$. From the (2.10), there are the following expresses:

$$
\begin{aligned}
2 T_{0}= & 6 k \epsilon^{2} P\left(\frac{r_{1}}{16 \pi P r_{1}^{2}+3 k\left(1-\epsilon^{2}\right)}+\frac{r_{2}}{16 \pi P r_{2}^{2}+3 k\left(1-\epsilon^{2}\right)}\right) \\
& +2\left(r_{1}+r_{2}\right) P-\frac{k}{8 \pi}\left(1 / r_{1}+1 / r_{2}\right), \\
0= & 6 k \epsilon^{2} P\left(\frac{r_{1}}{16 \pi P r_{1}^{2}+3 k\left(1-\epsilon^{2}\right)}-\frac{r_{2}}{16 \pi P r_{2}^{2}+3 k\left(1-\epsilon^{2}\right)}\right) \\
& +2\left(r_{1}-r_{2}\right) P-\frac{k}{8 \pi}\left(1 / r_{1}-1 / r_{2}\right) .
\end{aligned}
$$


For the simplicity we define the parameter as $y \equiv \frac{16 \pi r_{+}^{2}}{k} P$, so there are

$$
y_{1} \equiv \frac{16 \pi r_{1}^{2}}{k} P, \quad y_{2} \equiv \frac{16 \pi r_{2}^{2}}{k} P
$$

at the boundary of the two-phase coexistence area. The above equations can be rewritten as

$$
\begin{aligned}
T_{0}= & \frac{y_{2} k}{16 \pi r_{2}} \times\left[\frac{[1+x]\left[y_{2} x-1\right]}{y_{2} x}+\frac{3 \epsilon^{2} x}{y_{2} x^{2}+3\left(1-\epsilon^{2}\right)}+\frac{3 \epsilon^{2}}{y_{2}+3\left(1-\epsilon^{2}\right)}\right], \\
0= & y_{2}^{3} x^{3}+y_{2}^{2} x\left[3\left(1+x^{2}\right)\left(1-\epsilon^{2}\right)+x\left(1-3 \epsilon^{2}\right)\right] \\
& +3 y_{2}\left(1-\epsilon^{2}\right)\left[1+3 x+x^{2}\right]+9\left(1-\epsilon^{2}\right)^{2},
\end{aligned}
$$

and the (3.1) becomes

$$
\begin{aligned}
& \frac{4 \pi r_{2} T_{0}}{k}\left(1+x-\frac{6\left(1-\epsilon^{2}\right) \ln x}{y_{2}(1-x)}\right) \\
& =\frac{y_{2}\left(1+x+x^{2}\right)}{3}+2-\frac{3\left(1-\epsilon^{2}\right)}{y_{2} x} .
\end{aligned}
$$

From the (2.9) and (2.10), we can obtain the critical parameters as

$$
y_{c}=\frac{2 \sqrt{3}-1}{3}, \quad \epsilon_{c}^{2}=\frac{4}{9}\left(1+\frac{2}{\sqrt{3}}\right) .
$$

For the critical point $(x=1)$, substituting $y=y_{c}$ and $\epsilon=\epsilon_{c}$ into the (2.10), the critical temperature $\left(T_{c}\right)$, pressure $\left(P_{c}\right)$ and horizon radius $\left(r_{c}\right)$ satisfy the following expressions

$$
\begin{aligned}
8 \pi r_{c} T_{c} & =\frac{4 \sqrt{3} k}{3} \\
16 \pi r_{c}^{2} P_{c} & =k y_{c}=\frac{(2 \sqrt{3}-1) k}{3} .
\end{aligned}
$$

For similarity, we introduce the new parameter $\xi=\frac{1-\epsilon^{2}}{1-\epsilon_{c}^{2}}$, the (3.6) can be transformed into

$$
\begin{aligned}
0= & \frac{3}{11}(15+8 \sqrt{3}) x^{3} y_{2}^{3}+y_{2} \xi\left(1+3 x+x^{2}\right)+\frac{1}{9}(15-8 \sqrt{3}) \xi^{2} \\
& +x y_{2}^{2}\left[\left(1+x+x^{2}\right) \xi-\frac{6}{11}(15+8 \sqrt{3}) x\right]
\end{aligned}
$$

Combining the (3.5) and (3.7), the expression related with $y_{2}$ and $x$ reads

$$
\begin{aligned}
& \frac{[1-x]\left(3\left(1+x+x^{2}\right) x y_{2}^{2}+18 x y_{2}-(15-8 \sqrt{3}) \xi\right)}{x y_{2}\left[9\left(1-x^{2}\right) y_{2}-2(15-8 \sqrt{3}) \xi \ln x\right]} \\
= & \frac{(1+x)\left(x y_{2}-1\right)}{x y_{2}}+\frac{27 x-(15-8 \sqrt{3}) \xi x}{9 x^{2} y_{2}+(15-8 \sqrt{3}) \xi}+\frac{27-(15-8 \sqrt{3}) \xi}{9 y_{2}+(15-8 \sqrt{3}) \xi} .
\end{aligned}
$$

It is obviously that for the given parameters $\xi$ and $\mathrm{k}$, the solutions of $y_{2}$ and $x$ can be obtained by solving the (3.11) and (3.12). Thus for the first-order phase transition of the HL AdS black hole with the given pressure, we can obtain the phase transition temperature $T_{0}$ and the horizons $\left(r_{1}\right.$ and $r_{2}$ ) of the black hole in two different phases by substituting the values 


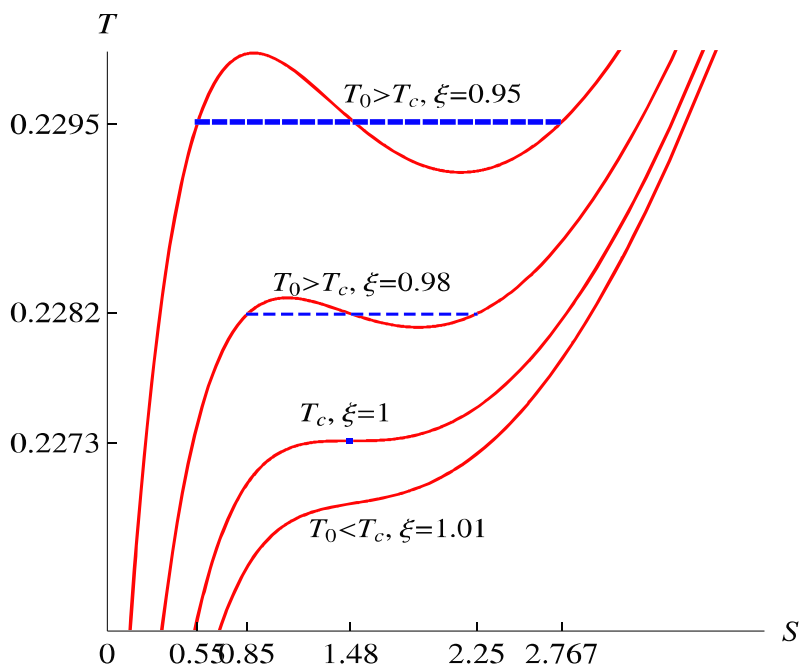

Fig. 1 The $T-S$ curves of the Horava-lifshitz AdS black hole with the different parameter values $\xi=\frac{1-\epsilon^{2}}{1-\epsilon_{c}^{2}}$. We set the pressure $P=0.1$ and $k=1$

of $\xi$ and $\mathrm{k}$ into the (3.4) and (3.7). The phase transition curves $T-S$ of the HL AdS black hole with different parameter values $\xi$ and the unchanged pressure $P=0.1$ are shown in Fig. 1.

From Fig. 1 we know that the phase transition of the HL AdS black hole will appear when the redefined parameter $\xi$ is less than one (namely, $\epsilon>\epsilon_{c}$ ), and the temperature $T_{0}$ is bigger than the critical one $T_{c}$. These behaviors are fully different from the other AdS black holes $[1,8,12,14,15,24,25,34,36,39,55]$. That maybe mean this system has the fully new structure from others and the physical mechanism of the phase transition is also unique.

For the first-order phase transition point of the HL AdS black hole with the given parameters $(k, \xi)$, because of $\frac{y_{1}}{y_{2}}=x^{2}$, there is $y=y_{2}$, or $y=y_{1}$, namely

$$
16 \pi r_{2}^{2} P=k y_{2}=k F_{2}, \quad \text { or } \quad 16 \pi r_{1}^{2} P=k y_{1}=k F_{1} \text {. }
$$

That indicates the first-order phase transition point is related with the horizon radius and pressure. As what we have known the pressure $F$ on the surface of the black hole's horizon reads: $F=A P=16 \pi r_{+}^{2} P=k P$. Thus the defined parameter $y$ stands for the pressure $F$ on the surface of the black hole's horizon. In the other words, the first-order phase transition point is only determined by the pressure $F$ on the surface of the black hole's horizon, that is different from the charged AdS black holes $[1,58,59]$. When the pressure $F$ on the surface of the black hole's horizon satisfies $16 \pi r_{c}^{2} P=k y_{c}$, the difference of the phase transition between this system and other charged AdS black holes will disappear.

From the (3.9) and (3.10), we can see that the critical temperature $T_{c}$ and pressure $P_{c}$ are not unique and are both related with the critical horizon radius. That means the parameters influencing the phase transition of the HL AdS black hole are different from other normal thermodynamic systems. With the above analyze, we find for the different values of $\xi$ in the HL AdS black hole with any given pressure, there may be a first-order phase transition, or a 
second-order, or nothing. Note that for the HL AdS black hole with a fixed parameter value $\left(\epsilon>\epsilon_{c}\right)$, there is a critical curve of phase transition, not only is a critical point.

\section{Discussions and Conclusions}

In this paper we mainly study the first-order phase transition of the HL AdS black hole by the construction of the equal-area law in $T-S$ diagram. With the above analyze, the characteristics of the thermodynamic property for the HL AdS black hole are summarized as: i) It is easy to see that from the (2.8) in the four-dimensional spacetime, the thermodynamical volume is zero when $y^{2}=9\left(1-\epsilon^{2}\right)$, while the horizon radius is not zero. That indicates there is the minimal horizon, which is related with the parameter $\epsilon$ and pressure $\mathrm{P}\left(16 \pi P r_{\min }^{2} / k=3\left(1-\epsilon^{2}\right)^{1 / 2}\right)$. ii) Since the location of horizon is independent with the temperature from the (3.11) and (3.12), the temperature is not the only factor to determine the phase transition, which is different from the other AdS black holes. iii) The phase transition is related with the pressure F on the surface of the black hole's horizon. In other words the phase transition of the four-dimensional HL AdS black hole with other fixed parameters $(k, \epsilon)$ is only determined by the pressure $\mathrm{F}$ on the surface of horizon.

Acknowledgments We would like to thank Prof. Zong-Hong Zhu and Meng-Sen Ma for their indispensable discussions and comments. This work was supported by the Natural Science Foundation of China (Grant No. 11475108 Grant No. 11705107, Grant No. 11475108, and Grant No. 12075143).

Open Access This article is licensed under a Creative Commons Attribution 4.0 International License, which permits use, sharing, adaptation, distribution and reproduction in any medium or format, as long as you give appropriate credit to the original author(s) and the source, provide a link to the Creative Commons licence, and indicate if changes were made. The images or other third party material in this article are included in the article's Creative Commons licence, unless indicated otherwise in a credit line to the material. If material is not included in the article's Creative Commons licence and your intended use is not permitted by statutory regulation or exceeds the permitted use, you will need to obtain permission directly from the copyright holder. To view a copy of this licence, visit http://creativecommons.org/licenses/by/4.0/.

\section{References}

1. Kubiznak, D., Mann, R.B.: P-v criticality of charged AdS black holes. JHEP 1207, 033 (2012). arXiv:1205.0559

2. Dolan, B.P., Kastor, D., Kubiznak, D., Mann, R.B., Traschen, J.: Thermodynamic volumes and isoperimetric inequalities for de Sitter black Holes. Phys. Rev. D 87, 10 (2013). arXiv:1301.5926

3. Gunasekaran, S., Kubiznak, D., Mann, R.B.: Extended phase space thermodynamics for charged and rotating black holes and Born-Infeld vacuum polarization. JHEP 11 arXiv:1208.6251 (2012)

4. S, B.-Y., Wang, Y.-Y., Li, N.: The Hawking-Page phase transitions in the extended phase space in the Gauss-Bonnet gravity. Eur. Phys. J. C 80, 305 (2020). arXiv:1905.07155

5. Wei, S.-W., Liu, Y.-X., Mann, R.B.: Ruppeiner geometry, phase transitions, and microstructure of charged ads black holes. Phys. Rev. D 100, 124033 (2019). arXiv:1909.03887

6. Sinovic, F., Mann, R.B.: Critical phenomena of born-infeld-de sitter black holes in cavities. JHEP 05, 136 (2019). arXiv:1904.04871

7. Altamirano, N., Kubizk, D., Mann, R.B., Sherkatghanad, Z.: Kerr-ads analogue of triple point and solid/liquid/gas phase transition. Class. Quant. Grav. 31 arXiv:1308.2672 (2014)

8. Anabalon, A., Gray, F., Gregory, R., Kubiznak, D., Mann, R.B.: Thermodynamics of charged, rotating, and accelerating black holes. JHEP 04, 096 (2019). arXiv:1811.04936

9. Cai, R.-G., Hu, Y.-P., Pan, Q.-Y., Zhang, Y.-L.: Thermodynamics of black holes in massive gravity. Phys. Rev. D 91, 2 (2015). arXiv:1409.2369 
10. Ge, X.-H., Ling, Y., Niu, C., Sin, S.-J.: Thermoelectric conductivities, shear viscosity, and stability in an anisotropic linear axion model. Phys. Rev. D 92, 10 (2015). arXiv:1412.8346

11. Cai, R.-G., Ruan, S.-M., Wang, S.-J., Yang, R.-Q., Peng, R.-H.: Action growth for AdS black holes. JHEP 09 arXiv:1606.08307 (2016)

12. Zhang, J.-L., Cai, R.-G., Yu, H.-W.: Phase transition and thermodynamical geometry of ReissnerNordstrom-AdS black holes in extended phase space. Phys. Rev. D 91, 4 (2015). arXiv:1502.01428

13. Zhao, R., Zhao, H.-H., Ma, M.-S., Zhang, L.-C.: On the critical phenomena and thermodynamics of charged topological dilaton AdS black holes. Eur. Phys. J. C 73 arXiv:1305.3725 (2013)

14. Zhao, R., Zhao, H.-H., Ma, M.-S., Zhang, L.-C.: On thermodynamics of charged and rotating asymptotically ads black strings. Adv. High Energy Phys. 7 (2013)

15. Hendi, S.H., Mann, R.B., Panahiyan, S., Eslam Panah, B.: van der Waals like behavior of topological AdS black holes in massive gravity. Phys. Rev. D 95 arXiv:1702.00432 (2017)

16. Hendi, S.H., Taghadomi, Z.S., Corda, C.: New aspect of critical nonlinearly charged black hole. Phys. Rev. D 97, 8 (2018). arXiv: 1803.10767

17. Hendi, S.H., Talezadeh, M.S., Armanfard, Z.: Phase transition of black holes in Branes-Dicke BornInfeld gravity through geometrical thermodynamics. Adv. High Energy Phys. arXiv:1709.00289 (2017)

18. Hendi, S.H., Panahiyan, S., Eslam Panah, B., Jamil, M.: A new prescription towards thermodynamic phase transition. Chin. Phys. C 43(11), 113106 (2019). arXiv:1706.07662

19. Hendi, S.H., Eslam Panah, B., Panahiyan, S., Talezadeh, M.S.: Geometrical thermodynamics and P-V criticality of black holes with power-law Maxwell field. Eur. Phys. J. C 77, 2 (2017). arXiv:1612.00721

20. Hendi, S.H., Panahiyan, S., Eslam Panah, B., Faizal, M., Momennia, M., Mehrab: Critical behavior of charged black holes in Gauss-Bonnet gravity's rainbow. Phys. Rev. D 94, 2 (2016). arXiv:1607.06663

21. Hendi, S.H., Tad, R.M., Armanfard, Z., Talezadeh, M.S.: Extended phase space thermodynamics and PV criticality: Brans-Dicke-Born-Infeld vs. Einstein-born-infeld-dilaton black holes. Eur. Phys. J. C 76, 5 (2016). arXiv:1511.02761

22. Hendi, S.H., Panahiyan, S., Panah, B.E., Armanfard, Z.: Phase transition of charged Black Holes in Brans-Dicke theory through geometrical thermodynamics. Eur. Phys. J. C 76, 7 (2016). arXiv: 1511.00598

23. Hendi, S.H., Sheykhi, A., Panahiyan, S., Eslam Panah, B.: Phase transition and thermodynamic geometry of Einstein-Maxwell-dilaton black holes. Phys. Rev. D 92, 6 (2015). arXiv:1509.08593

24. Dehyadegari, A., Sheykhi, A., Montakhab, A.: Critical behavior and microscopic structure of charged AdS black holes via an alternative phase space. Phys. Lett. B 768, 235 (2017). arXiv:1607.05333

25. Zou, D.-C., Yue, R.-H., Zhang, M.: Reentrant phase transitions of higher-dimensional AdS black holes in dRGT massive gravity. Eur. Phys. J. C 77, 4 (2017). arXiv:1612.08056

26. Xu, J.-F., Cao, L.-M., Hu, Y.-P.: P-v criticality in the extended phase space of black holes in massive gravity. Phys. Rev. D 91, 12 (2015). arXiv:1506.03578

27. Hendi, S.H., Behnamifard, H., Bahrami-Asl, B.: Energy-dependent topological anti-de Sitter black holes in Gauss-Bonnet Born-Infeld gravity. PTEP 3 arXiv:1804.10519 (2018)

28. Hobrecht, H., Hucht, A.: Anisotropic scaling of the two-dimensional Ising model II: Surfaces and boundary fields. SciPost Phys. 8, 032 (2020). arXiv:1805.00369

29. Maeda, H., Svarc, R., Podolsky, J.: Decreasing entropy of dynamical black holes in critical gravity. JHEP 06 arXiv:1805.00026 (2018)

30. Meng, K.: Hairy black holes of Lovelock-Born-Infeld-scalar gravity. Phys. Lett. B 784 arXiv:1804.10951 (2018)

31. Brihaye, Y., Hartmann, B.: Critical phenomena of charged Einstein-Gauss-Bonnet black holes with charged scalar hair. Class. Quant. Grav. 35, 17 (2018). arXiv:1804.10536

32. Dayyani, Z., Sheykhi, A., Dehghani, M.H., Hajkhalili, S.: Critical behavior and phase transition of dilaton black holes with nonlinear electrodynamics. Eur. Phys. J. C 78, 2 (2018). arXiv: 1709.06875

33. Ovgn, A.: $P-v$ criticality of a specific black hole in $f(R)$ gravity coupled with Yang-Mills field. Adv. High Energy Phys. arXiv:1710.06795 (2018)

34. Bhattacharya, K., Majhi, B.R.: Thermogeometric description of the van der Waals like phase transition in AdS black holes. Phys. Rev. D 95, 10 (2017). arXiv:1702.07174

35. Zhang, L.-C., Zhao, R.: The entropic force in Reissoner-Nordstrom-de Sitter spacetime. Phys. Lett. B 797, 134798 (2019)

36. Majhi, B.R., Samanta, S.: P-v criticality of AdS black holes in a general framework. Phys. Lett. B 773 arXiv:1609.06224 (2017)

37. Johnson, C.V.: Large $\mathrm{n}$ phase transitions, finite volume, and entanglement entropy. JHEP 047, 1403 (2014). arXiv:1306.4955

38. Caceres, H., Nguyen, P.H., Pedraza, J.F.: Holographic entanglement entropy and the extended phase structure of STU black holes. JHEP 184, 1509 (2015). arXiv:1507.06069 
39. Nguyen, P.H.: An equal area law for holographic entanglement entropy of the ads-RN black hole. JHEP 12, 139 (2015). arXiv:1508.01955

40. Zeng, X.-X., Zhang, H.-B., Li, L.-F.: Phase transition of holographic entanglement entropy in massive gravity. Phys. Lett. B 756, 170 (2016). arXiv:1511.00383

41. Lan, S.-Q., Li, G.-Q., Mo, J.-X., Xu, X.-B.: New phase transition related to the black holes topological charge. Adv. High Energy Phys. 8270265 arXiv:1804.06652 (2019)

42. Zeng, X.-X., Hu, X.-Y., Gao, Y.-J.: Holographic thermalization in the background with conformal anomaly corrected black holes. Sci. Sin-Phys. Mech. Astron. 46, 060401 (2016)

43. Wu, S.-Y., Ling, L.: Holographic phase transition for a black hole in the background with dark energy. Sci. Sin-Phys. Mech. Astron. 47, 060602 (2017)

44. Liu, H., von Hausegger, S., Naselsky, P.: Towards understanding the Planck thermal dust models. Phys. Rev. D 95, 10 (2017). arXiv:1705.05530

45. Gunasekaran, S., Mann, R.B., Kubiznak, D.: Extended phase space thermodynamics for charged and rotating black holes and Born-Infeld vacuum polarization. JHEP 11, 110 (2012). arXiv:1208.6251

46. Fujimori, T., Kimura, T., Nitta, M., Ohashi, K.: 2d partition function in $\Omega$-background and vortex/instanton correspondence. JHEP 12, 110 (2015). arXiv: 1509.08630

47. Kord Zangeneh, M., Dehyadegari, A., Sheykhi, A., Mann, R.B.: Microscopic origin of black hole reentrant phase transitions. Phys. Rev. D 97, 8 (2018). arXiv:1709.04432

48. Wei, S.-W., Liu, Y.-X.: Triple points and phase diagrams in the extended phase space of charged GaussBonnet black holes in AdS space. Phys. Rev. D 90, 4 (2014). arXiv:1402.2837

49. Hennigar, R.A., Mann, R.B., Tjoa, E.: Superfluid black holes. Phys. Rev. Lett. 118, 021301 (2017)

50. $\mathrm{Xu}, \mathrm{W} .: \lambda$ phase transition in Horava gravity. Adv. High Energy Phys. 2175818 arXiv:1804.03815 (2018)

51. Ma, M.-S., Wang, R.-H.: Peculiar $P-V$ criticality of topological Horava-Lifshitz black holes. Phys. Rev. D 96, 2 (2017). arXiv:1707.09156

52. Li, T.-J., Qi, Y.-H., Wu, Y.-L., Zhang, Y.-L.: Topological charged black holes in generalized HoravaLifshitz gravity. Phys. Rev. D 90, 12 (2014). arXiv:1405.4457

53. Lu, H., Mei, J.-W., Pope, C.N.: Solutions to horava gravity. Phys. Rev. Lett. 103, 091301 (2009). arXiv:0904.1595

54. Cai, R.-G., Cao, L.-M., Ohta, N.: Topological black holes in Horava-Lifshitz gravity. Phys. Rev. D 80, 024003 (2009)

55. Mo, J.-X., Zeng, X.-X., Li, G.-Q., Jiang, X., Liu, W.-B.: A unified phase transition picture of the charged topological black hole in Horava-Lifshitz gravity. JHEP 10, 056 (2013). arXiv:1404.2497

56. Cai, R.-G., Cao, L.-M., Ohta, N.: Thermodynamics of black holes in Horava-Lifshitz gravity. Phys. Lett. B 679, 504 (2009). arXiv:0905.0751

57. Majhi, B.R., Roychowdhury, D.: Phase transition and scaling behavior of topological charged black holes in Horava-Lifshitz gravity. Class. Quant. Grav. 29, 245012 (2012). arXiv:1205.0146

58. Wei, S.-W., Liu, Y.-X.: Insight into the microscopic structure of an ads black hole from a thermodynamical phase transition. Phys. Rev. Lett. 115, 111302 (2015). arXiv: 1502.00386

59. Wei, S.-W., Liu, Y.-X., Robert, B.M.: Repulsive interactions and universal properties of charged anti-de sitter black hole microstructures. Phys. Rev. Lett. 123, 071103 (2019). arXiv:1906.10840

Publisher's Note Springer Nature remains neutral with regard to jurisdictional claims in published maps and institutional affiliations. 